\title{
Virtual Simulation on Ultrasound Non-destructive Testing of Solid Rocket Motor
}

\author{
${ }^{1}$ Dachun Zhang, ${ }^{2}$ Xiaochun Luo and ${ }^{1}$ Shiying Zhang \\ ${ }^{1} D^{1} 602$, Department Six, Second Artillery Engineering University, Xi'an, China \\ ${ }^{2}$ The $3^{\text {rd }}$ Institute, Equipment Academy of the Second Artillery, Beijing, China \\ Email: Dachzhang@yahoo.com.cn
}

\begin{abstract}
Conventionally, ultrasound-based non-destructive testing (UNDT) is employed to examine the solid rocket motor (SRM) during its surveillance and maintenance. To avoid training with real equipment, virtual UNDT training system has been developed. To enhancement training effect with high immersion, a novel scheme to generate ultrasound signal according to the preset detected material is proposed to visually demonstrate the process of UNDT approximately. In this scheme, the virtual ultrasound signal is obtained based on the linear scattering model and a material defect model. To corroborate the proposed method, signals from three kinds of typical regions were respectively simulated. It shows that the proposed scheme is able to distinguish the defect status of the regions as real UNDT both in quality and in quantity.

Index Terms - Virtual ultrasound non-destructive testing ( $v$ UNDT); Virtual Reality (VR); Solid rocket motor (SRM)
\end{abstract}

\section{Introduction}

For the solid rocket motor (SRM), training with real equipment is conventionally impermissible. To be convinced of the performance status of the equipment, an alternate way for technical training is to develop a virtual, immersive training system, as virtual reality (VR) technique advances greatly in recent years with the progress of the techniques of computer, spatial data processing, sensors, human-computer interaction, etc.

For an important item of SRM health inspection, ultrasound-based non-destructive testing (UNDT) is necessary to be simulated and integrated in the virtual training system of SRM. Since defects in SRM were not known in advance, and the mode that ultrasound acts on the detected material was complex, virtual UNDT of SRM was conventionally implemented in an over-simplified way. In this method, some priori fault modes were firstly acquired to form a database and integrate in the virtual system. When the trained maintainers operating on the virtual scene, one of the modes was called and displayed. Such a manner has two obvious limitations. On the one hand, the status of defect cannot be set or adjusted randomly, so it cannot fully reflect real equipment defect. On the other hand, there are no strict relationship between the virtual operation and the displayed fault scene. Furthermore, the fault modes to be acquired must be limited, so the efficiency for virtual system-based training will be degraded.

To overcome the above-mentioned limitations of conventional method, based on linear elasticity ultrasound scattering model, a virtual UNDT ( $v$ UNDT) method is proposed in this paper. Different from the conventional method, the proposed method can obtain the ultrasound echo of far-field region by constructing defect model to interact with the ultrasound scattering model according to a randomly preset defect mode.

\section{Theory}

UNDT is based on the difference of the ultrasound signal waveform when the ultrasound is transmitted back from the region with difference defect status. Therefore, it is necessary to generate ultrasound signal according to the defect within the detected region for an immersive $v$ UNDT system. However, the mathematic description of ultrasound spread is complex ${ }^{[1,}$ 2].

To simplify the problem, the generation of the ultrasound echo in this paper is implemented with two modules, the ultrasound scattering model and the detected material model. The ultrasound scattering model is decided by the ultrasound pulse radiated from the ultrasound machine. The material model is decided by the macro- and micro-structure of the detected material. Then, the resulted ultrasound echo is the interaction of the two models.

Based on above simplification, the ultrasound echo for nondestructive testing can be expressed with the following equation ${ }^{[3]}$

$s(x, y, z)=f(x, y, z) * p(x, y, z)+n(x, y, z)$

where, $s(x, y, z)$ is the echo signal; $f(x, y, z)$ is the spatial point spread function (psf), which characterizes the ultrasound machine; $p(x, y, z)$ is the ultrasound scattering function ( $\mathrm{sf}$ ) used to describe the scattering character of the scanned material; $x, y, z$ are the spatial coordinates in three regular directions, respectively; $*$ is the convolution operation and $n(x, y, z)$ represent a uncorrelated random noise due to electronic disturbance.

\section{Simulation Methods}

Because of the strong randomization of the detected material in micro structure, it will become much complex to consider the ultrasound scattering process in 3-dimensional space with the theoretical model in (1). Therefore, the two models are simplified in virtual simulation. 


\section{A. Ultrasound Scattering Funtion}

To simplify the problem, and also consider the time efficiency, a linear elastic ultrasound scattering model [3] is introduced in this paper. The model can be described as follow.

Firstly, the detected material is regarded as a serial of uniformly distributed scatterers in the 3-dimensional space. Then, for each scatterer it has a norm-distributed scattering strength which is expressed as a preset random number with its mean value of 0.5 and standard difference of 0.2 . The spatial distribution density of the scatterers is set to be 20 per millimeter, which satisfies the ultrasound reflection character of the material ${ }^{[4]}$.

\section{B. Ultrasound Point Spread Function}

The ultrasound psf is simulated as a spatial function which is axially symmetry about the central axis of the transducer. In mathematics, it can be expressed in the following ${ }^{[5]}$

$$
p(x, r)=p_{x}(x) p_{r}(r)
$$

where, $p_{x}(x)$ and $p_{r}(r)$ are the component of the psf in the axial direction and the radial direction, respectively.

The axial component is simulated as a Gaussian modulated cosine function, which is formulated as ${ }^{[3,6]}$

$$
p_{x}(x)=\frac{1}{\sqrt{2 \pi} \sigma} e^{-\left(\frac{x^{2}}{2 \sigma^{2}}\right)} \cos \left(k_{0} x\right)
$$

where, $k_{0}=\frac{2 \pi}{\lambda_{0}}$ is the wave number of the ultrasound pulse and used to characterize the center frequency of the ultrasound; $\lambda_{0}$ is the wave length of the ultrasound pulse and $\sigma$ is a correlation length.

To be consistent with practical ultrasound transducer used for UNDT of the SRM, the center frequency of the axial component is set to $5-\mathrm{MHz}$ with band width of $60 \%$. The waveform of the axial component is simulated as shown in figure 1(a).

The radial component of the psf is simulated as a cosine function in the following form ${ }^{[5,6]}$

$$
p_{r}(r)=\cos ^{2}\left(\frac{\pi r}{2 R}\right)
$$

where, $r$ is the distance from a point in the sectional plane to the axis,; $R$ is the radius of the transducer, which is set to $20-\mathrm{mm}$ to be consistent with the real UNDT equipment. Figure 1(b) shows the simulated waveform of the radial component.

The comprehensive psf of the system is obtained by substituting (3) and (4) into (2). Figure 1(c) shows the comprehensive waveform of the spatial psf. It can be found the waveform of the psf presented as Gaussian modulated cosine vibration in the axial direction, and, in the radial direction, as descending from center to the circumference with a cosine relationship.

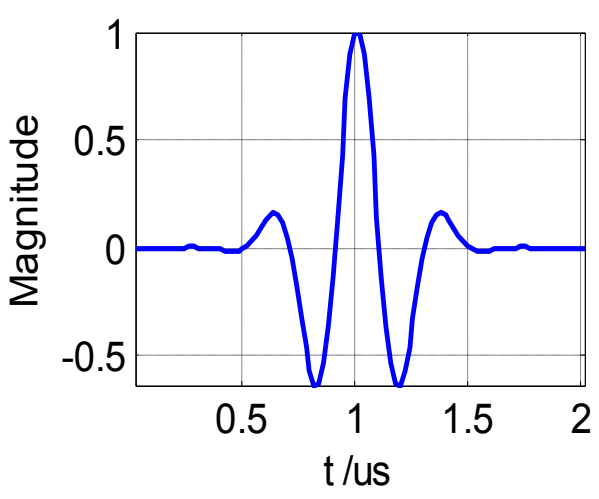

(a) Axial waveform

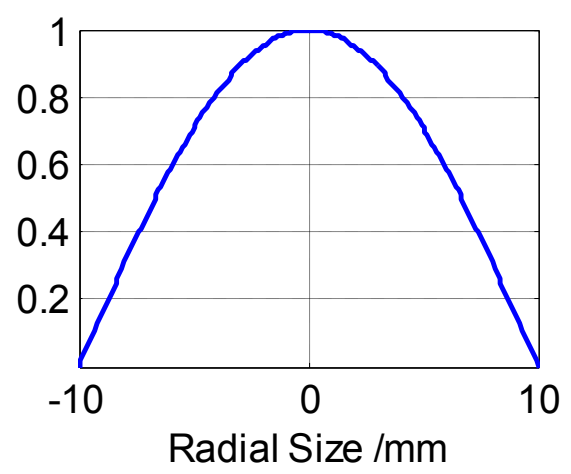

(b) Radial waveform

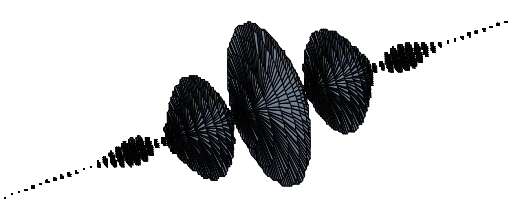

(c) spatial waveform

Figure 1. waveform of the point spread function

\section{Defect Model}

To verify the proposed scheme, a shell material model for a detected region with a preset defect mode is constructed. As shown in Figure 2, the simulated material is modeled as a cuboid structure with a spatial size of $250 \times 250 \times 5 \mathrm{~mm}^{3}$. In the center of the structure top-viewed, a circular region with a radius of $50-\mathrm{mm}$ is simulated as a defect. The defect region is located from the top surface of the simulated material with a depth of $3.5-\mathrm{mm}$. To consider the material using in the SRM, the simulated material is supposed to be steel, and the speed of ultrasound in the material is set to $5000-\mathrm{m} / \mathrm{s}$. 


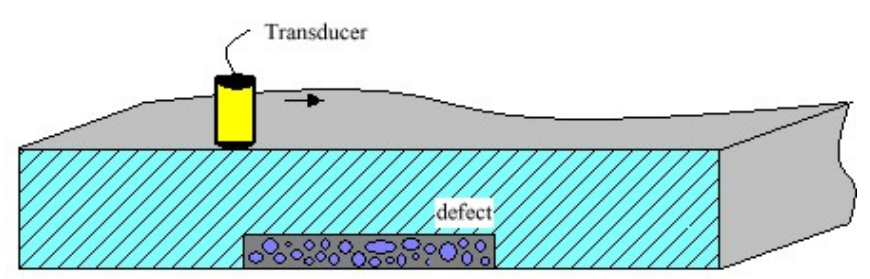

Figure 2. The cutaway view of the defect detection model

In the scattering function, material defect is characterized as a region with strong scattering edge, and it is implemented by increasing the scattering strength of the scatters on the edge. This paper focuses on the ultrasound defect-detection method of multi-reflection, which is one of the basic modes for SRM defect-detection at cylinder section to find the shape and size of the de-bond region. For such a mode, much of the ultrasound energy is lose in the non-defect region because there is no strong scattering interface. As a result, the magnitude of ultrasound decreased quickly in the echo. On the contrary, when in the region with defects, the energy of ultrasound radiated into the material is reflected and then generates multi-reflection due to the strong scattering at the interface between the defect region and normal region. Ultimately, the magnitude of ultrasound echo decreases slow relative to the normal region.

\section{Results}

To evaluate the proposed method, the waveforms of ultrasound echo from typical regions and the corresponding defect size measurement were simulated, respectively.

\section{D. waveform of the simulated ultrasound signal}

For ultrasound defect-detection with multi-refection, the defect locating and its size measurement are both according to the height of the echo signal ${ }^{[7]}$, as stated in above part. Therefore, ultrasound from three kinds of typical regions were simulated and compared in this part. The three kinds of regions, non-defect region (NDR), half-defect region (HDR) and full-defect region (FDR), are roughly described as follow respectively. For each kind of region, a circular region with radius of $10 \mathrm{~mm}$, which is consistent to the size of the simulated transducer, is chosen to compare the simulated echo.

(1) The NDR is referred to the region where no defect is within the cylinder of ultrasound beam. In this paper, the region to represent the NDR is centered at the point $(50-\mathrm{mm}$, 50-mm top viewed).

(2) The HDR is referred to the region where the ultrasound beam partly gets across the defect region. In this paper, the region to represent the HDR is centered at the point $(75-\mathrm{mm}$, 125-mm top viewed).

(3) The FDR is referred to the region where the defect is full of the whole region the ultrasound beam spreads. In implementation, the region to represent the FDR is centered at the point of (125-mm, 125-mm top viewed).

The spatial shapes of the three kinds of typical regions are illustrated in Figure 3, as indicated with the circles. For each kind of region, its location viewed from the top of the simulated defect model is demonstrated in Figure 3(a). In the figures, the defect region is represented with a dark-mesh circular.

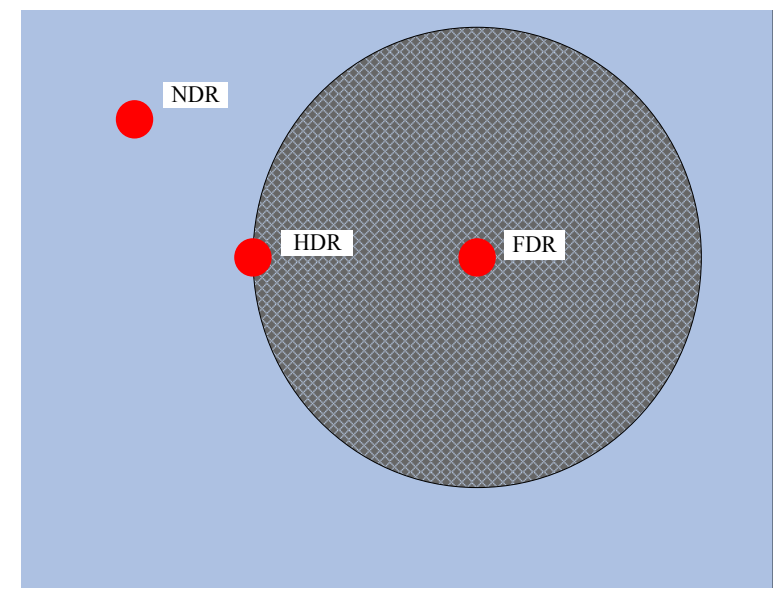

(a) The distribution of the regions (top-viewed)

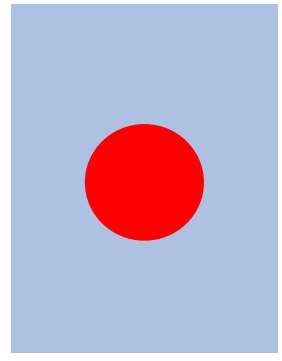

(b) NDR

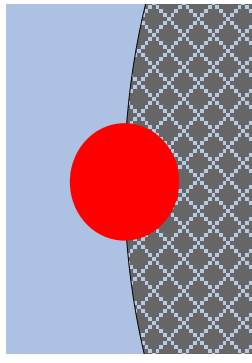

(c) HDR

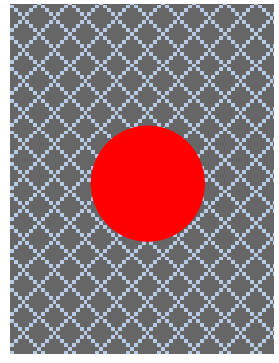

(d) FDR
Figure 3. Three kinds of typical regions for simulation (within the circles)

From Figure 4, it can be found that there exists evident difference in the magnitude of the signal waveforms for the three different regions.

(1) For the echo from the NDR (Figure 3b), the magnitude of the simulated ultrasound echo decreases quickly, as shown in Figure 4(a). In this region, the rocket shell is well conglutinated to its inner structures. So when reached this interface, ultrasound can radiate forward into the inner structure, and only a small proportion of energy reflects back to the shell. As a result, the energy of the ultrasound echo drops quickly.

(2) For the echo form the FDR (Figure 3d), as shown in Fig 4c, the magnitude of the echo signal attenuates evidently slower than that from the NDR (Figure 4a). It can be explained as follows. In this region, when ultrasound reaches the interface between the norm shell and the defect region, most of the ultrasound energy will be reflected back to the shell and further forms multi-reflection because of the obvious difference in sound impendence on the interface. In this circumstance, energy lose is mainly due to the attenuation in the shell. 


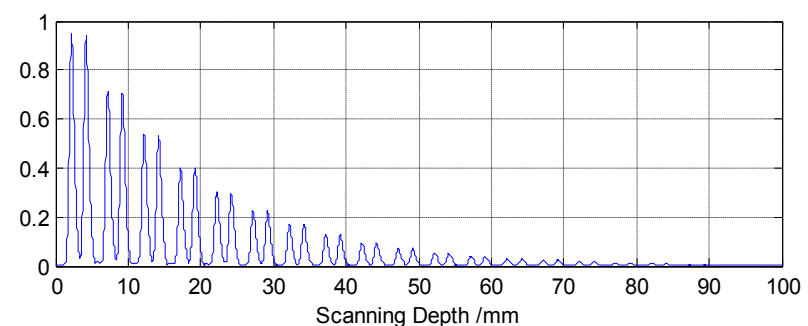

(a) Waveform for NDR

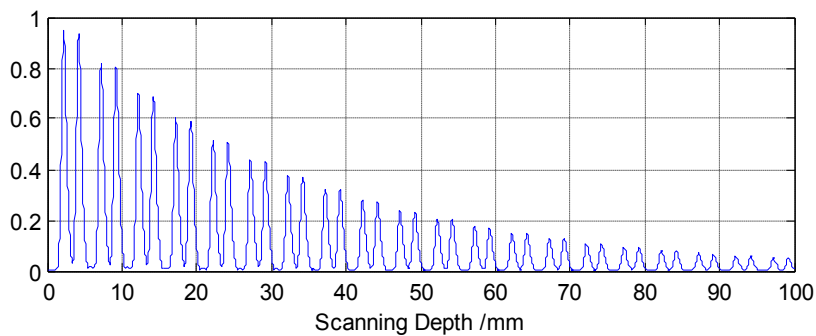

(b) Waveform for HDR

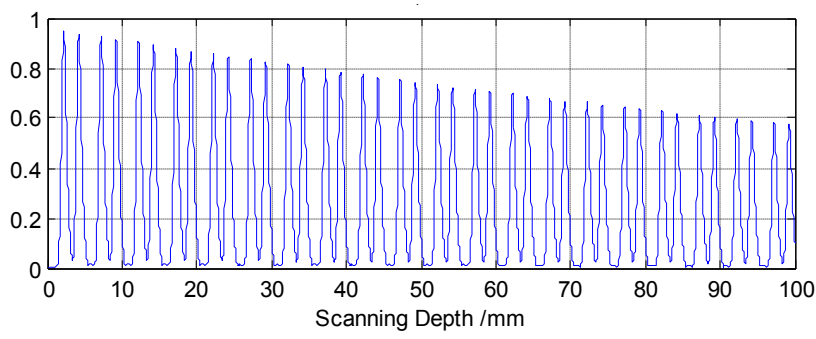

(c) Waveform for FDR

Figure 4. Ultrasound echo Waveform from the three typical regions, respectively.

(3) For the echo from the HDR, the attenuation presents as a transition of that in the NDR and the FDR. For a specified scanning depth, the height of the simulated echo is higher than that from NDR, and lower than that from FDR.

In conclusion, the height of the echo in a specified scanning depth reflects the proportion of the defect region in the radiating range of the simulated transducer. Therefore, by means of the height of the echo magnitude in a predefined depth, the edge of the defect can be defined and further to be used to measure the size of the defect.

\section{E. Defect size measurement}

For the model in Figure 2, simulation was implemented in an interval of every $1 \mathrm{~mm}$ along the transversal diameter of the defect region. The height of echo in the scanning depth of 60 $\mathrm{mm}$ was considered for each location, and the resulted curve for these points from a typical simulation was shown in Figure 5 .

It can be found that the height of the echo has evident difference for the three kinds of regions. This is consistent to the theoretical analysis above.

The size of the defect was obtained by measure the width of curve at the half-height of the maximum value and the minimum value, as shown by the arrow line in Fig 5 . With 50 times independent simulation, the measured width of the defect was $92.6 \pm 0.3 \mathrm{~mm}$, which is some smaller than that in the model $(100-\mathrm{mm})$. The accuracy of the obtained defect size is related to the measurement scheme. However, the proposed method is robust even the echo is noisy, as verified with more simulations.

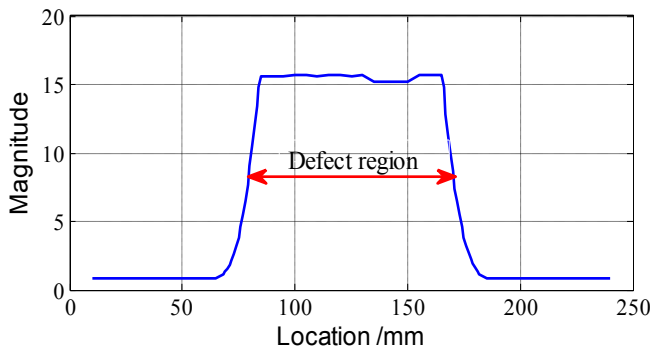

Figure 5. Scheme of virtual defect size measurement

\section{Conclusion}

In this paper, a virtual ultrasound non-destructive testing method for the cylinder section of SRM is proposed based on the linear ultrasound scattering model. Results from simulation shows, the proposed method can not only simulate the ultrasound waveform qualitatively according to the preset defect mode, but also realize the process of the defect quantification. Comparing to the traditional virtual scheme, the proposed strategy has more consistency no mater in the procedure or on the result, which is help to enhance the immersion of the virtual training system.

Note that only a preliminary study of vUNDT was presented in this paper with its emphasis on the feasibility of the proposed scheme. To integrate the scheme with the virtual maintainance system, more works need to do, such as defect setting and producing, detection of virtual operation, evaluation of the virtual operation, etc.

\section{References}

[1] Morse P M and Ingard K U. Theoretical Acoustics. New York: McGraw-Hill, 1968.

[2] Pierce A. Acoustics : an introduction to physical principles and applications. New York: McGraw Hill, 1986.

[3] Dachun Zhang, Siyuan Zhang, Mingxi Wan and Supin Wang. A fast tissue stiffness-dependent elastography for HIFU-induced lesions inspection[J]. Ultrasonics, 2011, 51(8): 857-869

[4] Wagner RF, Smith JM, Lopez H. Statistics of speckle in ultrasound Bscans [J]. IEEE Trans Sonics Ultrason, 1983, 30(3): 156-163

[5] Jing Bai, Chuxiong Ding, Jianwen Luo and Ping He, Estimation and reduction of decorrelation effect due to tissue lateral displacement in elastography, IEEE Trans Ultras., Ferr. Freq. Contrl., 2002, 49(5): 541 549.

[6] Dachun Zhang, Performance Optimizing methods and Experimental investigation in Elastography[D]. Xi'an: Xi'an Jiaotong University ph.D disertation, 2012

[7] Chen $\mathrm{C} \mathrm{H}$, Ultrasonic and advanced methods for non-destructive testing and material characterization, London: World Scientific Publishing Co Pte ltd., 2007. 